\title{
INVESTIGATION OF INTERMITTENCY IN MAGNETOHYDRODYNAMICS AND SOLAR WIND TURBULENCE: SCALE-DEPENDENT KURTOSIS
}

\author{
Minping Wan ${ }^{1}$, Kareem T. Osman ${ }^{1}$, William H. Matthaeus ${ }^{1}$, and Sean Oughton ${ }^{2}$ \\ ${ }^{1}$ Bartol Research Institute and Department of Physics and Astronomy, University of Delaware, Newark, DE 19716, USA; mpwan@bartol.udel.edu \\ 2 Department of Mathematics, University of Waikato, Hamilton 3240, New Zealand \\ Received 2011 September 4; accepted 2011 October 2; published 2011 December 22
}

\begin{abstract}
The behavior of scale-dependent (or filtered) kurtosis is studied in the solar wind using magnetic field measurements from the $A C E$ and Cluster spacecraft at 1 AU. It is also analyzed numerically with high-resolution magnetohydrodynamic spectral simulations. In each case the filtered kurtosis increases with wavenumber, implying the presence of coherent structures at the smallest scales. This phase coupling is related to intermittency in solar wind turbulence and the emergence of non-Gaussian statistics. However, it is inhibited by the presence of upstream waves and other phase-randomizing structures, which act to reduce the growth of kurtosis.
\end{abstract}

Key words: magnetohydrodynamics (MHD) - solar wind - turbulence

Online-only material: color figures

\section{INTRODUCTION}

In the last 20 years, intermittency in the solar wind has been widely analyzed by using satellite observations of solar wind fluctuations (Burlaga 1991; Marsch \& Tu 1997; Sorriso-Valvo et al. 1999; Voros et al. 2002, 2006; Koga et al. 2007). These studies have added to the growing body of evidence that the solar wind provides a natural laboratory for investigations of magnetohydrodynamic (MHD) turbulence (Matthaeus \& Goldstein 1982; Biskamp 2003). Indeed, many features of MHD turbulence have been revealed in the solar wind (Tu \& Marsh 1995; Goldstein et al. 1995; Bruno \& Carbone 2005).

The phenomenon of intermittency is related to the emergence of small-scale coherent structures that are responsible for enhanced dissipation, and in steady state these structures cause departures from self-similarity and the emergence of characteristic scaling laws (Frisch 1995). Classically, the notion of intermittency can be discussed in the inertial range, and equivalently, in the dissipation range. Intermittency has been deeply investigated in both hydrodynamic and MHD turbulence (Frisch 1995; Sreenivasan \& Antonia 1997; Biskamp 2003). Here we will employ a method based on scale-dependent kurtosis to characterize intermittency in MHD and in the solar wind. This method is related to several others that we will review below, and we will argue that it provides a clear perspective on the relationship between scales, coherency, and higher order statistics that may be useful in observational and numerical studies.

To motivate our methodology, we recall that perhaps the most fundamental approach to studying intermittency is to examine the non-Gaussian behavior of the probability density function (PDF) of the dissipation function. However, adopting Kolmogorov's refined similarity hypothesis (KRSH; Kolmogorov 1962; Oboukhov 1962) allows the local averages of dissipation to be related to the increments of velocity, with both quantities remaining as random variables. This approach leads to the familiar connection of intermittency to the PDF of the increments of the velocity in hydrodynamics (Anselmet et al. 1984). This scale-dependent non-Gaussianity is also observed in the MHD primitive variables (velocity and magnetic field) in simulations (Biskamp et al. 1990), theoretical models (Carbone 1994), and in solar wind data (Burlaga 1993; Marsch \& Tu 1994;
Sorriso-Valvo et al. 1999). We note that particularly in the case of the solar wind, the scale-dependent non-Gaussianity may be observed either in the inertial range or as a property of the intermittent solar source. Here we are concerned only with smallscale non-Gaussianity. Increments are computed for separation $r$ and the non-Gaussianity of the PDFs is found to increase with decreasing $r$. A useful way to quantify this is the kurtosis of the increments, which increases with decreasing $r$ (see, e.g., Voros et al. 2002; Bruno et al. 2003), as well as the normalized higher order moments, which also increase at smaller separations (Dudok de Wit \& Krasnosel'skikh 1996). A related methodology is to quantify intermittency based on the sequence of $p$ th moments (integer $p$ ) of the increment PDFs, which then lead to power-law scalings $\sim r^{\zeta}$ with increment separation where the power-law indices $\zeta(p)$ depend on moment order $p$. The behavior of these scaling exponents is connected to various fractal and multi-fractal models (Frisch et al. 1978; Burlaga 1991; She \& Leveque 1994; Grauer et al. 1994; Politano \& Pouquet 1995; Horbury \& Balogh 1997). The physical content of these models lies in the sensitivity of the higher order moments to concentrations of dissipation and, through the $\mathrm{KRSH}$, to large values of the increments. Thus, the higher order scalings are associated with the presence of coherent structures, usually assumed to be generated by the turbulence cascade.

While the mathematical framework of the multi-fractal scalings does not lend itself to a transparent interpretation in terms of coherent structures, other approaches, based on direct examination of local structure, have improved understanding of the same issues.

For some time there have been suggestions that intense structures of electric current density, appearing during MHD turbulent relaxation (Matthaeus \& Montgomery 1980; Frisch et al. 1983; Matthaeus \& Lamkin 1986; Carbone et al. 1990), are real-space manifestations of intermittency. For even longer, and with little apparent connection, magnetic directional discontinuities (DDs) have been studied in the interplanetary medium (Burlaga 1968; Burlaga \& Ness 1969; Tsurutani \& Smith 1979; Neugebauer 2006). Rather than a consequence of turbulence these "DDs" or "D-sheets" have typically been viewed as convecting or propagating solutions of ideal MHD equations, possibly broadened by diffusion. Only relatively recently has the 
potential connection with turbulence been emphasized. This is based on comparison with distributions of the normalized magnetic field increments, a statistic called the partial variance of increments (PVI) that links magnetic field gradients to turbulence intermittency, and comparison of waiting time distributions in turbulence simulations and solar wind observations (Greco et al. 2008, 2009).

There has also been considerable success in applying filtering methods such as wavelets (Farge 1992) to identify the most intermittent events in spacecraft data (Veltri \& Mangeney 1999). The approach here is to find wavelet amplitudes that exceed a chosen multiple of the average amplitude, defining these as "intermittent events." Just as in the simulation studies (Matthaeus \& Montgomery 1980; Greco et al. 2009), one finds that sheet-like structures contribute substantially to the observed intermittency and that after the removal of these events (Veltri \& Mangeney 1999) the remaining fluctuations better follow Gaussian scalings of higher order moments. The intermittency events themselves (rather than the remaining nearGaussian fluctuations) have also been studied employing a wavelet approach to identify "events" (Bruno et al. 2001).

Another very interesting approach to studying effects associated with intermittency is the method of phase coherence (Hada et al. 2003; Koga \& Hada 2003; Koga et al. 2007). The basis of this idea is that signals containing coherent structures must include fluctuations that add constructively at the positions of the structures. Therefore, a non-Gaussian intermittent signal can be compared with both a phase-randomized signal and a perfectly phase-coherent signal to produce a measure of coherency and intermittency, called the phase coherence index (PCI). Combined with wavelet low-pass filtering (Koga \& Hada 2003) the PCI method has been shown to produce results that are consistent with small-scale intermittency due to coherent structures, which however are mainly found at frequencies lower than the ion gyro-frequency.

A final class of methods is based on computing the normalized fourth-order moment, the kurtosis. While large kurtosis signals non-Gaussianity and often super-Gaussian tails on the PDF of fluctuations, the analysis becomes much more revealing when it is applied selectively to ranges of length scales. In this way, the influence of small-scale coherent structures in producing intermittency can be detected. The usual way that this has been implemented (Voros et al. 2002; Bruno et al. 2003; Bolzan 2005; Chian \& Miranda 2009) is to compute the kurtosis of the increments of the fluctuations. Then letting the increment lag become smaller, one expects increased kurtosis if smallscale coherent structures are present. It is interesting to note that it has recently been shown that the PCI method performs similarly to a method based on computing the kurtosis of the increments of the vector magnitudes (Chian \& Miranda 2009). The kurtosis of increments method is clearly related to the defining characteristic of intermittency as given by Frisch (1995): a signal is considered intermittent if its high-pass filtered kurtosis is increasing with increasing cutoff frequency. However, as it is based on increments, and not a high-pass filter, the connection is heuristic (or at least complex).

Here we modify the kurtosis method to employ the high-pass filtered kurtosis method directly, examining both solar wind data and simulation data. While the present approach is connected conceptually to the several families of methods described above, the filtered kurtosis method addresses the Frisch definition directly while maintaining a close connection to the moments of the PDF of fluctuations. Furthermore, as it is based on a sharp
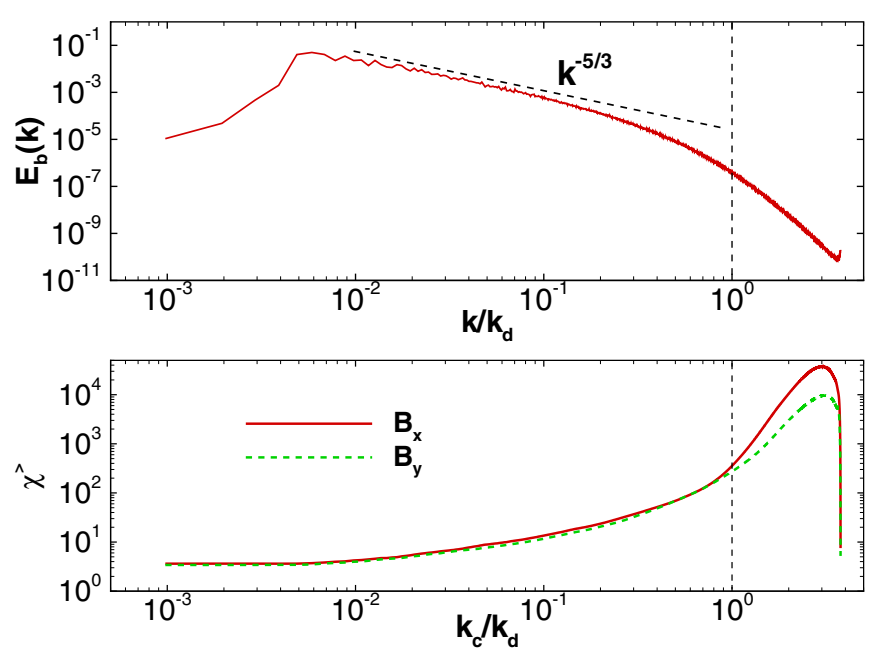

Figure 1. Magnetic energy spectrum (top) and filtered kurtosis of the magnetic field $\chi^{>}$(bottom) from an $8192^{2}$ MHD simulation. For comparison, a $k^{-5 / 3}$ power law is also plotted in the top panel. The Kolmogorov dissipation scale is indicated by the vertical dashed lines.

(A color version of this figure is available in the online journal.)

Fourier high-pass filter, this approach permits an exact identification of the scales at which the measured coherence resides.

In the following sections we calculate the scale-dependent kurtosis of the magnetic field using Cluster and $A C E$ data, and compare the results to those obtained from high-resolution twodimensional (2D) MHD simulations.

\section{SCALE-DEPENDENT KURTOSIS}

The scale-dependent (or filtered) kurtosis, $\chi^{>}\left(k_{\mathrm{c}}\right)$, is defined as the kurtosis of a high-pass filtered variable (e.g., magnetic field $\boldsymbol{B}$ ); that is, Fourier components of the variable with wavenumbers $k<k_{\mathrm{c}}$ are zeroed (Frisch 1995; Wan et al. 2009, 2010). This quantity measures a characteristic that is very close to what Frisch (1995) discussed as the defining property of spatial intermittency. In Wan et al. (2010) it is demonstrated using numerical simulations that the scale-dependent kurtosis of electric current density $\chi_{j}^{>}\left(k_{\mathrm{c}}\right)$ and vorticity $\chi_{\omega}^{>}\left(k_{\mathrm{c}}\right)$ are sharply increasing functions of the high-pass cutoff wavenumber $k_{\mathrm{c}}$ when a run is well resolved.

To get a general idea of scale-dependent kurtosis, in Figure 1 we show the (high-pass) filtered kurtosis of magnetic fluctuations together with the magnetic energy spectrum from an $8192^{2}$ incompressible MHD simulation (see Section 3.2 for simulation details). As is well known, the global (unfiltered) kurtosis of magnetic fluctuations is close to 3 and the PDFs of the components of $\boldsymbol{b}$ are also close to Gaussian. However, Figure 1 clearly shows a non-Gaussian feature of the magnetic field, namely that its filtered kurtosis increases sharply for higher cutoff wavenumber $k_{\mathrm{c}}$. Moreover, the rate of increase of $\chi^{>}\left(k_{\mathrm{c}}\right)$ continues to increase well into the dissipation range.

In Figure 2 we plot the same quantities, but now calculated from a $4 \mathrm{hr}$ interval of Cluster data (detailed description of data selection will be given in the next section). $\mathrm{A} k^{-5 / 3}$ inertial range is evident in the magnetic energy spectrum. Comparing the two figures, one observes that the general behavior of $\chi^{>}\left(k_{\mathrm{c}}\right)$ for these Cluster data is very similar to that from the 2D MHD simulation.

We note that in order to accomplish a comparison of simulation and solar wind data sets, it is advantageous to normalize the relevant quantities in a physically meaningful way. 

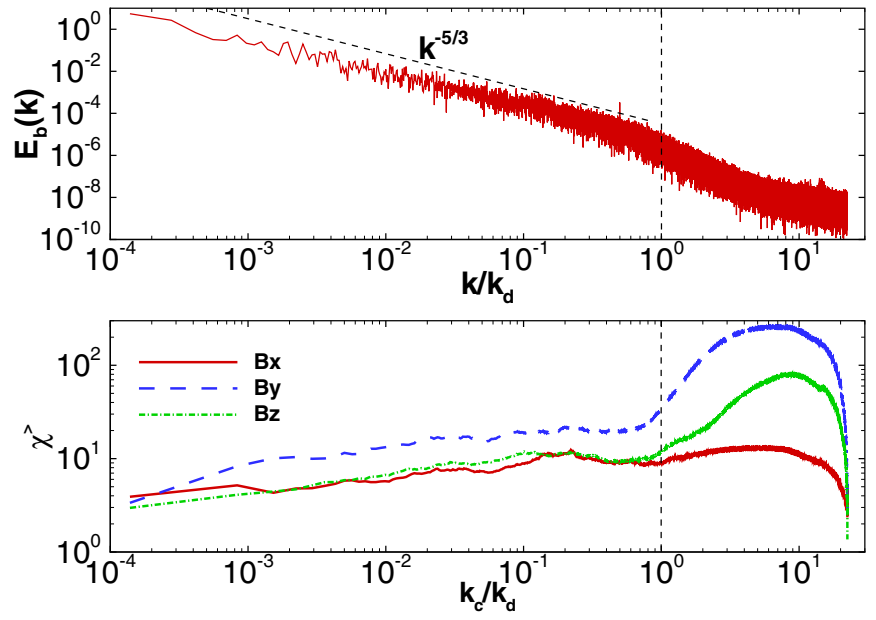

Figure 2. Magnetic energy spectrum (top) and filtered kurtosis $\chi^{>}$(bottom) from a $4 \mathrm{hr}$ interval of Cluster data. The vertical dashed lines correspond to the estimated Kolmogorov dissipation scale of $0.5 \mathrm{~Hz}$, where frequencies higher than this are generally considered to be in the dissipation range.

(A color version of this figure is available in the online journal.)

In earlier studies of waiting times between detections of coherent structures, normalization of length scales to the corresponding correlation scales was preferred (Greco et al. 2008, 2009), in part because some of the data sets did not extend to very high wavenumber. Here we will instead normalize to computed or estimated values of a scale connected approximately with the short-wavelength end of the inertial range. This was empirically found to work well for present purposes because the scale-dependent kurtosis becomes sensitive, at large values of the cutoff wavenumber, to the small amount of energy in the remaining signal.

With this motivation, the analysis of both simulation and solar wind data sets will be scaled to the computed or estimated Kolmogorov dissipation scale $\eta_{\mathrm{d}}$, as in Figures 1 and 2. For hydrodynamics, $\eta_{\mathrm{d}} \equiv 1 / k_{\mathrm{d}}=(\nu / \epsilon)^{1 / 4}$, where $\nu$ and $\epsilon$ are the kinematic viscosity and energy dissipation rate, respectively. An MHD Kolmogorov scale can be defined in an analogous way. In general, the inertial range lies at wavenumbers $k \eta_{\mathrm{d}} \ll 1$ and the dissipation range at $k \eta_{\mathrm{d}} \geqslant 1$. For solar wind data, we choose $k_{\mathrm{d}} \eta_{\mathrm{d}} \sim 1$ to correspond to the scale at which magnetic field spectra typically steepen, departing from the inertial range power-law behavior that extends to lower spacecraft frequencies. Various studies (e.g., Leamon et al. 1998) have shown that this "breakpoint" often occurs near the ion inertial scale, which through the Taylor frozen-in hypothesis is often found near $0.5 \mathrm{~Hz}$ at $1 \mathrm{AU}$ in the spacecraft frame. For simplicity we use this value for normalization of solar wind data sets. For simulations we employ the hydrodynamic formula.

In the analysis below, an additional step in the procedure gives improved comparisons. This is motivated by the fact that the short-wavelength signals in simulations and solar wind data have distinctive complications. Simulations have truncation error and possibly a small reflection from the cutoff wavenumber. The solar wind data have various errors associated with instrument accuracy, averaging, and telemetry limitations, etc. In order that the high wavenumber comparison avoid unintentionally comparing these spurious factors, we use a low-pass filter to cut off both types of data at their respective dissipation scales. All subsequent analyses employ data that are first low-pass filtered in this way (i.e., before spectra or scale-dependent kurtosis are calculated).

\section{DATA SELECTION AND ANALYSIS}

\subsection{Solar Wind Data Selection}

We analyze five years of magnetic field measurements (2001-2005) using $1 \mathrm{~s}$ and 22.4 sample per second resolution data respectively from the $A C E$ (Smith et al. 1998) and Cluster 1 (Balogh et al. 2001) spacecraft at 1 AU. The magnetic field vectors are in geocentric solar ecliptic (GSE) coordinates, with the $x$-axis along the Sun-Earth line and the $z$-axis toward ecliptic north. A convenient ensemble is obtained by dividing the data into $4 \mathrm{hr}$ intervals. This duration is long enough to contain several correlation times (Matthaeus et al. 2005), but short enough to avoid large-scale inhomogeneities. Within each interval, the kurtosis of each magnetic field component must lie within the range $2.8-4.0$ to ensure the global behavior is approximately Gaussian. In order to maintain statistical stationarity, intervals are removed from the ensemble if they contain heliospheric current sheet crossings. Intervals are also discarded if the amount of missing or bad data exceeds $5 \%$, since this reduces the statistical robustness of any computed quantities. The remaining intervals are examined visually for transient events. Ten intervals that best match our criteria are selected from each spacecraft ensemble, and these constitute the solar wind data set for this present study.

\subsection{MHD Simulations}

To provide numerical examples, we also carried out a series of simulations of decaying incompressible 2D MHD turbulence. Use of 2D simulation is a reasonable approach given that solar wind fluctuations are widely held to be quasi-2D (see, e.g., Bieber et al. 1996). Computationally, the reduced dimensionality affords the possibility of better spatial resolution and higher Reynolds numbers. Expressed in terms of the vorticity $\omega=(\nabla \times v) \cdot \hat{z}$ and the vector potential $a$, the 2D MHD equations are

$$
\begin{gathered}
\frac{\partial \omega}{\partial t}=\nabla \cdot[\boldsymbol{b} j-\boldsymbol{v} \omega]+v \nabla^{2} \omega \\
\frac{\partial a}{\partial t}=-\boldsymbol{v} \cdot \nabla a+\mu \nabla^{2} a .
\end{gathered}
$$

Here, $\boldsymbol{v}$ is the fluid velocity, $\boldsymbol{b}=\nabla a \times \hat{z}$ is the magnetic field, and $j=-\nabla^{2} a$ is the electric current density; these are functions of $x, y$, and $t$. The viscous and resistive dissipation coefficients are, respectively, $\nu$ and $\mu$ and are equivalent to reciprocal Reynolds numbers with the normalization employed.

Equations (1) and (2) are solved numerically in a $2 \pi$-periodic box using a Fourier spectral method with phaseshift dealiasing (Patterson \& Orszag 1971). The time integration is a second-order Runge-Kutta method. All runs are performed with $v=\mu$ and are unforced. Initial $(t=0)$ spectra of $v$ and $\boldsymbol{b}$ are chosen proportional to $\left[1+\left(k / k_{0}\right)^{8 / 3}\right]^{-1}$, within a band of wavenumber $k=|\boldsymbol{k}|$; phases are assigned using Gaussian random numbers. The initial kinetic and magnetic energies are equal, $E_{v}=\left\langle|\boldsymbol{v}|^{2}\right\rangle / 2=0.5, E_{b}=\left\langle|\boldsymbol{b}|^{2}\right\rangle / 2=0.5$.

Two simulations are reported on herein, with respective resolutions of $4096^{2}$ and $8192^{2}$. For the largest $8192^{2}$ run, the initial Reynolds number is 20,000. Table 1 summarizes the run parameters.

\section{RESULTS}

The average magnetic energy spectrum is computed separately for the $A C E$ and Cluster data sets. To average the spectra, 

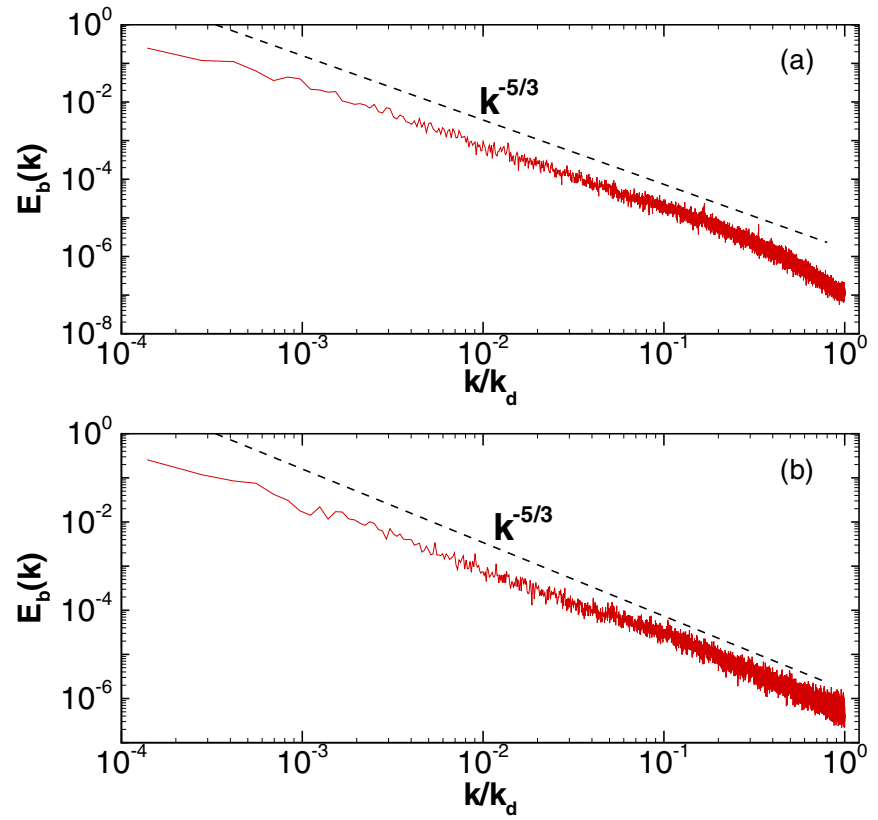

Figure 3. Magnetic energy spectrum averaged over the entire (a) $A C E$ and (b) Cluster data sets. A low-pass filter has removed frequencies above $0.5 \mathrm{~Hz}$, and any spikes at harmonics of the spacecraft spin frequency have also been removed. A $k^{-5 / 3}$ power law is plotted for comparison (dashed).

(A color version of this figure is available in the online journal.)

Table 1

Parameters for the Simulations

\begin{tabular}{lccccc}
\hline \hline Run & Grid & $R e$ & $k_{\max }$ & $k_{\mathrm{d}}$ & $\frac{k_{\max }}{k_{\mathrm{d}}}$ \\
\hline 1 & $4096^{2}$ & 8333 & 1931 & 633 & 3.05 \\
2 & $8192^{2}$ & 20000 & 3862 & 1169 & 3.30 \\
\hline
\end{tabular}

Notes. Here $k_{\mathrm{d}}$ means the maximum value of the time-dependent dissipation wavenumber $k_{\mathrm{d}}(t) . k_{\max }$ is the maximum retained wavenumber in the simulation. The initially excited Fourier modes have wavenumbers $k \in[5,20]$.

first each individual energy spectrum value is normalized by the corresponding total magnetic energy:

$$
E_{b}^{\prime}(k)=E_{b}(k) / \sum_{k} E_{b}(k) .
$$

A low-pass filter is applied to remove frequencies above $0.5 \mathrm{~Hz}$. As stated above, this is to focus our analysis on the inertial range and to eliminate effects such as measurements that approach the noise floor of the instruments. Figure 3 shows the resulting averaged energy spectra, where any spikes at harmonics of the spacecraft spin frequency have been removed by rescaling the Fourier components.

We compute the scale-dependent kurtosis of each magnetic field component for all the intervals within the solar wind data set. Figure 4 shows the results obtained from two of the intervals within the Cluster ensemble. In both cases, the kurtosis is around 3 at the largest scales. This suggests that globally the magnetic field components are statistically approximately Gaussian, which is consistent with expectations (Marsch \& Tu 1994; Bruno et al. 2003, 2007; Koga et al. 2007). However, the filtered kurtosis of the interval associated with Figure 4(a) increases essentially monotonically as the cutoff wavenumber $k_{\mathrm{c}}$ increases, until $k_{\mathrm{c}}$ reaches close to the maximum allowed wavenumber. This behavior of $\chi^{>}\left(k_{\mathrm{c}}\right)$ is considered a definition of intermittency (Frisch 1995) and is consistent with the
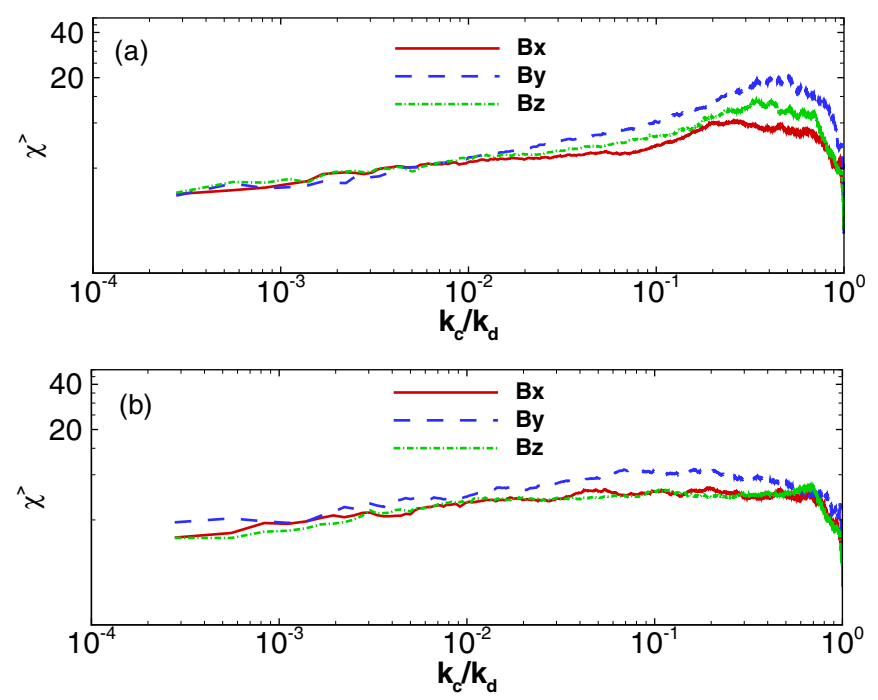

Figure 4. Scale-dependent kurtosis for each magnetic field component. The panels correspond to two distinct intervals from the Cluster data set. Panel (a) displays signatures of intermittency, whereas the interval for panel (b) contains upstream waves and much weaker intermittency features.

(A color version of this figure is available in the online journal.)

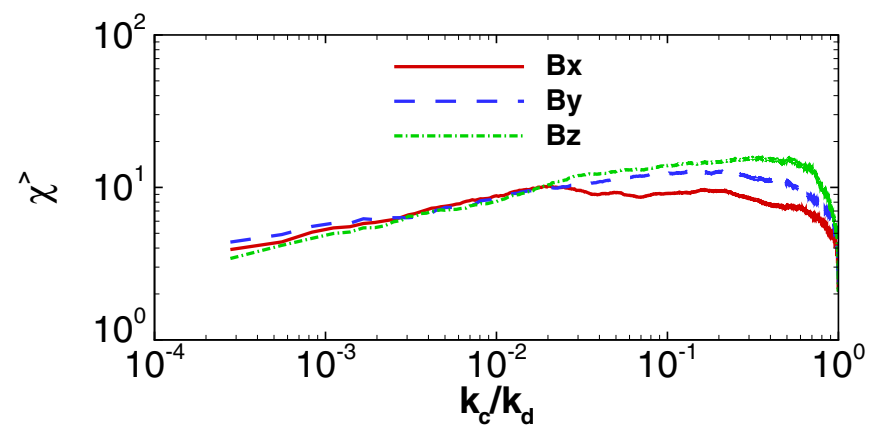

Figure 5. Scale-dependent kurtosis averaged over the 10 Cluster intervals.

(A color version of this figure is available in the online journal.)

magnetic field vector being an intermittent quantity. This too is consistent with results previously obtained in studies using a variety of techniques (Marsch \& Tu 1994; Bruno et al. 2003, 2007; Koga et al. 2007). Scale-dependent kurtosis provides a slightly different perspective, maintaining direct contact with both increment statistics and coherent structures. The steady rise in kurtosis implies the presence of coherent structures at the smallest scales since these are sources of intermittency in MHD turbulence, which are described by non-Gaussian statistics.

Figure 4(b) shows only a slight rise in kurtosis with increasing $k_{\mathrm{c}}$, which is consistent with the traditional view of an approximately Gaussian magnetic field. This conflicts with the results in Figure 4(a), despite both intervals being selected according to the same criteria. Further examination of the time series and associated spectrograms, obtained from the CIS instrument (Rème et al. 2001), reveals the presence of upstream waves in the Figure 4(b) interval. Indeed, the scale-dependent kurtosis of all the intervals in our solar wind data set is ordered by the extent of upstream waves within the time series: phase-randomizing structures impede the growth of kurtosis. Hence, our results support the interpretation of an intermittent magnetic field with phase-coherent structures at small scales. It appears that only when intervals are contaminated with upstream waves is their intermittent character hidden. Figure 5 shows the scale-dependent kurtosis, averaged over our entire Cluster data set, for each 


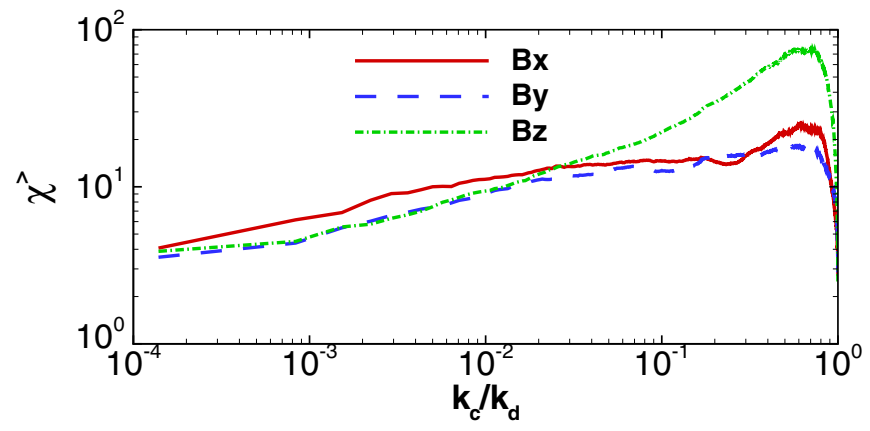

Figure 6. Scale-dependent kurtosis averaged over the $10 A C E$ intervals.

(A color version of this figure is available in the online journal.)
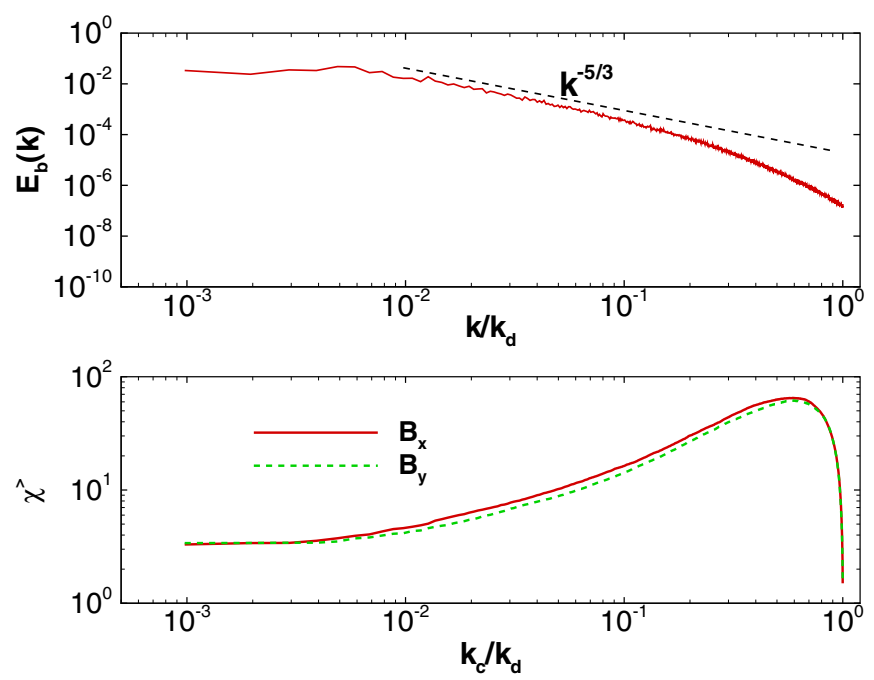

Figure 7. Averaged magnetic energy spectrum (top) and averaged scaledependent kurtosis of each magnetic field component (bottom) from an $8192^{2}$ MHD simulation. The averaging is performed over 100 data lines parallel to the $x$-axis.

(A color version of this figure is available in the online journal.)

magnetic field component. The trends with $k_{\mathrm{c}} / k_{\mathrm{d}}$ are similar to those seen in the "clean" interval of Figure 4(a), indicating that the majority of intervals within the data set confirm the presence of coherent structures and MHD turbulence intermittency (even though the Cluster spacecraft orbit makes it difficult to obtain completely uncontaminated intervals of solar wind data).

In comparison with Cluster, the $A C E$ spacecraft data set contains fewer upstream waves. Therefore, a stronger scaledependent kurtosis response is expected. Figure 6 shows $\chi^{>}$, averaged over our $A C E$ data set, for each magnetic field component. The intermittency signature is indeed greater, providing yet more evidence of coherent structures at small scales.

We now turn to examining data from the MHD simulations. It would be straightforward to calculate the filtered kurtosis using data from the whole simulation domain. However, to facilitate comparison with the observational results just presented, we instead only use data from straight line cuts through the domain - thereby simulating a spacecraft trajectory. Specifically, we first extract data from lines parallel to the $x$-axis and then use a low-pass filter to remove all scales smaller than the dissipation scale before we calculate the scale-dependent kurtosis.

In the top panel of Figure 7 we show the averaged magnetic energy spectrum, obtained by averaging 100 spectra each associated with a different line of data points. There is over a decade of $k^{-5 / 3}$ inertial range scaling. As noted in the previous
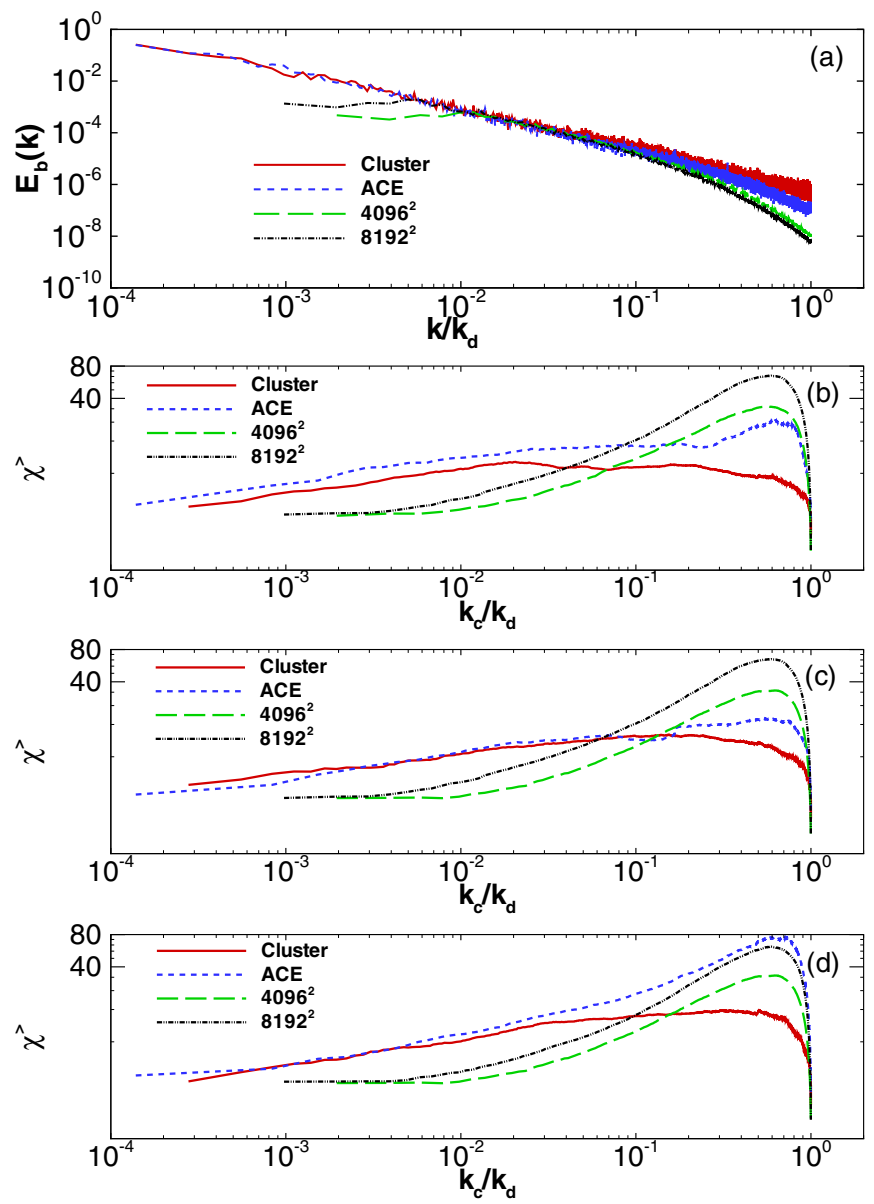

Figure 8. Averaged magnetic energy spectrum (a) and the scale-dependent kurtosis of magnetic field components (bottom three panels, $x, y, z$ components, respectively) from Cluster data, $A C E$ data, and simulation data. Note that for the simulations, the same $y$ component results are plotted in both panes (c) and (d) since the 2D simulations lack a $z$ component.

(A color version of this figure is available in the online journal.)

paragraph, the dissipation range scales were filtered out (i.e., Fourier components at scales smaller than the Kolmogorov scale were zeroed) before the individual "line" spectra were calculated. In the bottom panel we plot the averaged scaledependent kurtosis of the two magnetic field components. As expected we notice that $\chi^{>}\left(k_{\mathrm{c}}\right)$ increases with larger $k_{\mathrm{c}}$. Comparing with Figure 1, one observes that the rate of increase of $\chi^{>}$is smaller in Figure 7 due to the removal of the smallest scales. We note the interesting feature that the extremely high filtered kurtosis values in Figure 1 are found deep in the dissipation range, indicating the presence of structures with very high phase coherence at very small scales. However, as emphasized by Sreenivasan \& Antonia (1997), very small scale dissipative structures have a persistent influence seen even in the inertial range. The contrast between Figures 1 and 7 exemplifies their point, with the filtered kurtosis method making it very evident.

In Figure 8, we summarize the results from Cluster data, $A C E$ data, and simulation data. Panel (a) displays the averaged magnetic energy spectrum for each case. To aid comparison, the smallest scales of all data sets are normalized to be the same. Panels (b)-(d) show the averaged scale-dependent kurtosis of the individual magnetic field components. However, because the simulation is in two dimensions, we plot the simulation results 
from the $b_{y}$ component in both panels (c) and (d). Note that the data sets used in constructing this figure are low-pass filtered at the dissipation scales.

\section{CONCLUSIONS}

In this paper, we employ the method of scale-dependent (or filtered) kurtosis to study intermittency both in MHD simulations and in solar wind data near 1 AU. This method is closely related, in a number of ways, to several other methods that have been employed in various studies examining intermittency in MHD and in the solar wind. A representative sampling of these other studies was reviewed in the Introduction. The method we adopted herein has the advantage that it is very close to Frisch's straightforward definition of intermittency. It maintains close connection to the physics of scale-dependent PDFs while also affording a simple interpretation in terms of the coherent structures that are observed across the scales of the turbulence.

We studied the behavior of scale-dependent kurtosis in the solar wind using magnetic field measurements from the $A C E$ and Cluster spacecraft at $1 \mathrm{AU}$. For comparison, scale-dependent kurtosis is also analyzed numerically with highresolution incompressible MHD spectral simulations. Scaledependent kurtosis increases with wavenumber, implying the presence of coherent structures at the smallest scales. This phase coupling is related to intermittency in solar wind turbulence and the emergence of non-Gaussian statistics. However, in the solar wind data, increase of scale-dependent kurtosis at high cutoff wavenumbers might be inhibited by the presence of upstream waves and other phase-randomizing structures, which act to reduce the growth of kurtosis. This method may provide a sensitive tool for detecting the presence of phase-randomizing effects at small scales, whether they may be due to numerical effects, such as inadequate simulation resolution (Wan et al. 2010) or instrument noise, or of physical origin, as in the case of kinetically excited but apparently phase-randomized upstream waves. Effects similar to this have also been studied using phase coherency methods (Koga \& Hada 2003; Koga et al. 2007).

Finally, we note that the similarity in the behavior of the scaledependent kurtosis in the normalized analysis of solar wind data and MHD simulation data adds to the growing evidence that the physics of solar wind turbulence is closely related to what is seen in strong MHD turbulence (Greco et al. 2008, 2009; Osman et al. 2011).

This research is supported in part by NSF Solar Terrestrial Program grant ATM-0539995, NSF SHINE grant ATM-0752135, and NASA Heliophysics grants NNX09AH79 and NNX09AG31G, and the Strategic Research Investment Fund at the University of Waikato.

\section{REFERENCES}

Anselmet, F., Gagne, Y., Hopfinger, E. J., \& Antonia, R. A. 1984, J. Fluid Mech., 140,63

Balogh, A., Carr, C. M., Acuña, M. H., et al. 2001, Ann. Geophys., 19, 1207

Bieber, J. W., Wanner, W., \& Matthaeus, W. H. 1996, J. Geophys. Res., 101, 2511
Biskamp, D. 2003, Magnetohydrodynamic Turbulence (Cambridge: Cambridge Univ. Press)

Biskamp, D., Welter, H., \& Walter, M. 1990, Phys. Fluids B, 2, 3024

Bolzan, M. J. A. 2005, Brazilian J. Phys., 35, 592

Bruno, R., \& Carbone, V. 2005, Living Rev. Sol. Phys., 2, 4

Bruno, R., D’Amicis, R., Bavassano, B., Carbone, V., \& Sorriso-Valvo, L. 2007, Planet. Space Sci., 55, 2233

Bruno, R., Carbone, V., Sorriso-Valvo, L., \& Bavassano, B. 2003, J. Geophys. Res., 108, 1130

Bruno, R., Carbone, V., Veltri, P., Pietropaolo, E., \& Bavassano, B. 2001, Planet. Space Sci., 49, 1201

Burlaga, L. F. 1968, Sol. Phys., 4, 67

Burlaga, L. F. 1991, J. Geophys. Res., 96, 5847

Burlaga, L. F. 1993, J. Geophys. Res., 98, 17467

Burlaga, L. F., \& Ness, N. F. 1969, Sol. Phys., 9, 467

Carbone, V. 1994, Ann. Geophys., 12, 585

Carbone, V., Veltri, P., \& Mangeney, A. 1990, Phys. Fluids A, 2, 1487

Chian, A. C.-L., \& Miranda, R. A. 2009, Ann. Geophys., 27, 1789

Dudok de Wit, T., \& Krasnosel'skikh, V. V. 1996, Nonlinear Process. Geophys., 3,262

Farge, M. 1992, Annu. Rev. Fluid Mech., 24, 395

Frisch, U. 1995, Turbulence (Cambridge: Cambridge Univ. Press)

Frisch, U., Pouquet, A., Sulem, P.-L., \& Meneguzzi, M. 1983, J. Mech. Theor. Appl. Numero Special, 191

Frisch, U., Sulem, P.-L., \& Nelkin, M. 1978, J. Fluid Mech., 87, 719

Goldstein, M. L., Roberts, D. A., \& Matthaeus, W. H. 1995, ARA\&A, 33, 283

Grauer, R., Krug, J., \& Marliani, C. 1994, Phys. Lett. A, 195, 335

Greco, A., Chuychai, P., Matthaeus, W. H., Servidio, S., \& Dmitruk, P. 2008, Geophys. Res. Lett., 35, L19111

Greco, A., Matthaeus, W. H., Servidio, S., Chuychai, P., \& Dmitruk, P. 2009, ApJ, 691, 111

Hada, T., Koga, D., \& Yamamoto, E. 2003, Space Sci. Rev., 107, 463

Horbury, T. S., \& Balogh, A. 1997, Nonlinear Process. Geophys., 4, 185

Koga, D., Chian, A. C.-L., Miranda, R. A., \& Rempel, E. L. 2007, Phys. Rev. E, 75, 046401

Koga, D., \& Hada, T. 2003, Space Sci. Rev., 107, 495

Kolmogorov, A. N. 1962, J. Fluid Mech., 13, 82

Leamon, R. J., Smith, C. W., Ness, N. F., Matthaeus, W. H., \& Wong, H. K. 1998, J. Geophys. Res., 103, 4775

Marsch, E., \& Tu, C.-Y. 1994, Ann. Geophys., 12, 1127

Marsch, E., \& Tu, C.-Y. 1997, Nonlinear Process. Geophys., 4, 101

Matthaeus, W. H., Dasso, S., Weygand, J. M., et al. 2005, Phys. Rev. Lett., 95, 231101

Matthaeus, W. H., \& Goldstein, M. L. 1982, J. Geophys. Res., 86, 6011

Matthaeus, W. H., \& Lamkin, L. 1986, Phys. Fluids, 29, 2513

Matthaeus, W. H., \& Montgomery, D. 1980, Ann. New York Acad. Sci., 357, 203

Neugebauer, M. 2006, J. Geophys. Res., 111, A04103

Oboukhov, A. M. 1962, J. Fluid Mech., 13, 77

Osman, K. T., Wan, M., Matthaeus, W. H., Breech, B., \& Oughton, S. 2011, ApJ, 741, 75

Patterson, G. S., \& Orszag, S. A. 1971, Phys. Fluids, 14, 2538

Politano, H., \& Pouquet, A. 1995, Phys. Rev. E, 52, 636

Rème, H., Aoustin, C., Bosqued, J. M., et al. 2001, Ann. Geophys., 19, 1303

She, Z.-S., \& Leveque, E. 1994, Phys. Rev. Lett., 72, 336

Smith, C. W., Acuna, M. H., Burlaga, L. F., et al. 1998, Space Sci. Rev., 86, 611

Sorriso-Valvo, L., Carbone, V., \& Veltri, P. 1999, Geophys. Res. Lett., 26, 1801

Sreenivasan, K. R., \& Antonia, R. A. 1997, Annu. Rev. Fluid Mech., 29, 435

Tsurutani, B. T., \& Smith, E. J. 1979, J. Geophys. Res., 84, 2773

Tu, C.-Y., \& Marsch, E. 1995, Space Sci. Rev., 73, 1

Veltri, P., \& Mangeney, A. 1999, in AIP Conf. Proc. 471, Solar Wind IX, ed. S. Habbal (Melville, NY: AIP), 543

Voros, Z., Jankovicova, D., \& Kovacs, P. 2002, Nonlinear Process. Geophys., 9, 149

Voros, Z., Leubner, M. P., \& Baumjohann, W. 2006, J. Geophys. Res., 111, A02102

Wan, M., Oughton, S., Servidio, S., \& Matthaeus, W. H. 2009, Phys. Plasmas, 16,080703

Wan, M., Oughton, S., Servidio, S., \& Matthaeus, W. H. 2010, Phys. Plasmas, 17,082308 